\title{
First report of modern pollen deposition in moss polsters in a semiarid area of Bahia, Brazil ${ }^{1}$
}

\author{
Daiane Alves dos Santos ${ }^{2}$, Luciene Cristina Lima e Lima ${ }^{3}$, Francisco de Assis Ribeiro dos Santos ${ }^{4}$ \\ and Francisco Hilder Magalhães e Silva ${ }^{2 \star}$
}

Received: May 20, 2015. Accepted: August 6, 2015

\begin{abstract}
Recent studies have shown that pollen analyses of natural substrates can produce data valuable for understanding the local pollen productivity and dispersal, deposition, and preservation potential of pollen grains. In this study, we aimed to acquire novel information about the dynamics and preservation of pollen in Caatinga environment through the palynological study of moss polsters. Samples of moss polsters in soil (MPS) and on rock (MPR) were collected from the Canudos Biological Station in the Bahia State (Brazil) and subjected to standard chemical treatments for the extraction of pollen residues. In total, 372 pollen types were recorded from the samples of which the taxonomical affinity of 140 was determined. The most represented families were Fabaceae (23 pollen types/16.42\%) and Asteraceae (12 pollen types/8.57\%). The MPS samples had a higher pollen concentration $\left(21,042.04\right.$ pollen grains $\left./ \mathrm{cm}^{2}\right)$ than the MPR samples $\left(7,829.35\right.$ pollen grains $\left./ \mathrm{cm}^{2}\right)$. On the other hand, the MPR samples had a greater diversity $(68.26 \%$ of the identified pollen types). Qualitative analysis showed that the plants of shrub and subshrub habits had the greatest representation among the pollen types (35.0\%). Overall, moss polsters proved to be excellent natural air pollen collectors in Caatinga environment, provided they had moist microhabitats for their development.
\end{abstract}

Keywords: Brazilian NE, moss, natural pollen trap, pollen rain, semiarid

\section{Introduction}

Aerobiology is the study of the release, retention, dispersion, deposition and atmospheric incidence of airborne particles such as pollen, spores and bacteria (Pathirane 1975). Fontana (2003) stated that the relationship between modern pollen and current vegetation and the establishment of modern analogies between them help in interpreting paleoenvironments. In this sense, recent studies have related modern pollen rain, which is the atmospheric dispersal and deposition of current pollen grains, to different types of vegetation from both natural and artificial collectors (e.g., lacustrine sediments, peat, soil, moss polsters).

According to Lazarova et al. (2006), the use of natural substrates in modern pollen rain studies has been an effective method for understanding pollen productivity, potential dispersion, deposition and preservation and has contributed to a more detailed reconstruction of paleoecological conditions in a particular area. For example, information on pollen grains in samples from soil surfaces of the Ceará State recently provided important support for paleoenvironmental interpretations of the ecological response mechanisms of a tropical rainforest microrefuge in northeastern Brazil to paleoclimatic changes that occurred in this region (Montade et al. 2014). Coincidentally, 2014 also saw the publication of the first study regarding the record of pollen rain in natural collectors for the Caatinga environment (Gomes et al. 2014). In this pioneering study, the authors demonstrated the importance of bromeliad tanks as pollen deposits for local and regional vegetation that are able to store and preserve palynomorphs, which together reveal important information about the physiognomic and ecological aspects of Caatinga vegetation.

Along these lines, the present study aimed to expand the knowledge of the dynamics of pollen grains in the Caatinga environment and generate unprecedented information on pollen rain records in moss polsters that are present in this type of environment to provide support for future paleoecological and paleoclimatic studies in the Brazilian semiarid region.

\footnotetext{
${ }^{1}$ Based on the Master's dissertation of the first Author

${ }^{2}$ Programa de Pós-Graduação em Biodiversidade Vegetal, Departamento de Educação, Universidade do Estado da Bahia, Campus VII, 48.970-000, Senhor do Bonfim, BA, Brazil

${ }^{3}$ Programa de Pós-Graduação em Biodiversidade Vegetal, Departamento de Ciências Exatas e da Terra, Universidade do Estado da Bahia, Campus II, 48.040-210, Alagoinhas, BA, Brazil

${ }^{4}$ Programa de Pós-Graduação em Botânica, Universidade Estadual de Feira de Santana, Feira de Santana, 44.031-460, BA, Brazil

*Corresponding author: hildermagalhaes@hotmail.com
} 


\section{Material and methods}

\section{Study area}

The present study was undertaken in the Canudos Biological Station (CBS) in the municipality of Canudos in northeastern Bahia State, Brazil (400 m a.m.s.l.; 09 ${ }^{\circ} 54^{\prime}$ S; $39^{\circ} 07^{\prime} \mathrm{W}$ ), as shown in Fig. 1. This region experiences average monthly temperatures ranging from $20.7^{\circ} \mathrm{C}$ to $26.8^{\circ} \mathrm{C}$, with the warmest period being from November to March and coinciding with the rainy season, and annual rainfall is generally less than $800 \mathrm{~mm}$ (SEI 2013). The dry period (April to October) is defined as the period when total monthly precipitation, in millimeters, is equal to or lower than double the mean monthly temperature, as expressed in degrees Celsius $(\mathrm{P} \leq 2 \mathrm{~T}$ ) (Bagnouls \& Gaussen 1962).

The local vegetation is highly xerophytic, with areas of open shrub vegetation and denser tree and shrub growth along riversides and in valleys. The herbaceous stratum is poorly developed in valleys but fares better on hillsides, although it flourishes in both environments during the rainy season. A floristic list of the CBS, produced by Silva (2007), included 194 species among 141 genera and 54 families and provided information concerning growth habits and flowering periods.

\section{Collection of moss polsters}

We first performed survey work to locate moss polsters by hiking in the CBS. In dry periods, moss polsters were found only in cavities or in the outer areas of sandstone caves that had water drips inside. Only during rainy periods were moss polsters found outside sheltered caves, though they were nevertheless found very near sandstone hills and on rocks with the same composition. Samples were collected from an area of the CBS named Gruta do Minadouro ( $40 \mathrm{~m}$ length, $5 \mathrm{~m}$ width, $28 \mathrm{~m}$ height, approximately). Outside the cave was found open vegetation with a predominance of shrubs, subshrubs and herbs, but many trees were also present. Three samples were collected in September 2003, during the dry period, and five samples were collected in March 2005, during the rainy period. The codes MPS and MPR were applied to moss polster collected from soil surfaces and rocky walls, respectively. Two points were sampled: one was inside the cave, with collections in 2003 (MPR1, MPR2, MPS1) and 2005 (MPS2, MPS3), and the other was outside ( $\left.09^{\circ} 56^{\prime} 45.3^{\prime \prime} \mathrm{S} 38^{\circ} 59^{\prime} 12.9^{\prime \prime} \mathrm{W}\right)$, with collections only in 2005 (MPR3, MPR4, MPR5). Any moss polters in soil samples or on rock samples were collected in close proximity to one another (approximately $2 \mathrm{~m}$ ), but the distance between moss polters samples in soil and moss polters on rock was approximately $20 \mathrm{~m}$. Moss samples were extracted in block shapes, using a stylus for cutting, and were then packaged in paper bags and stored in a freezer.

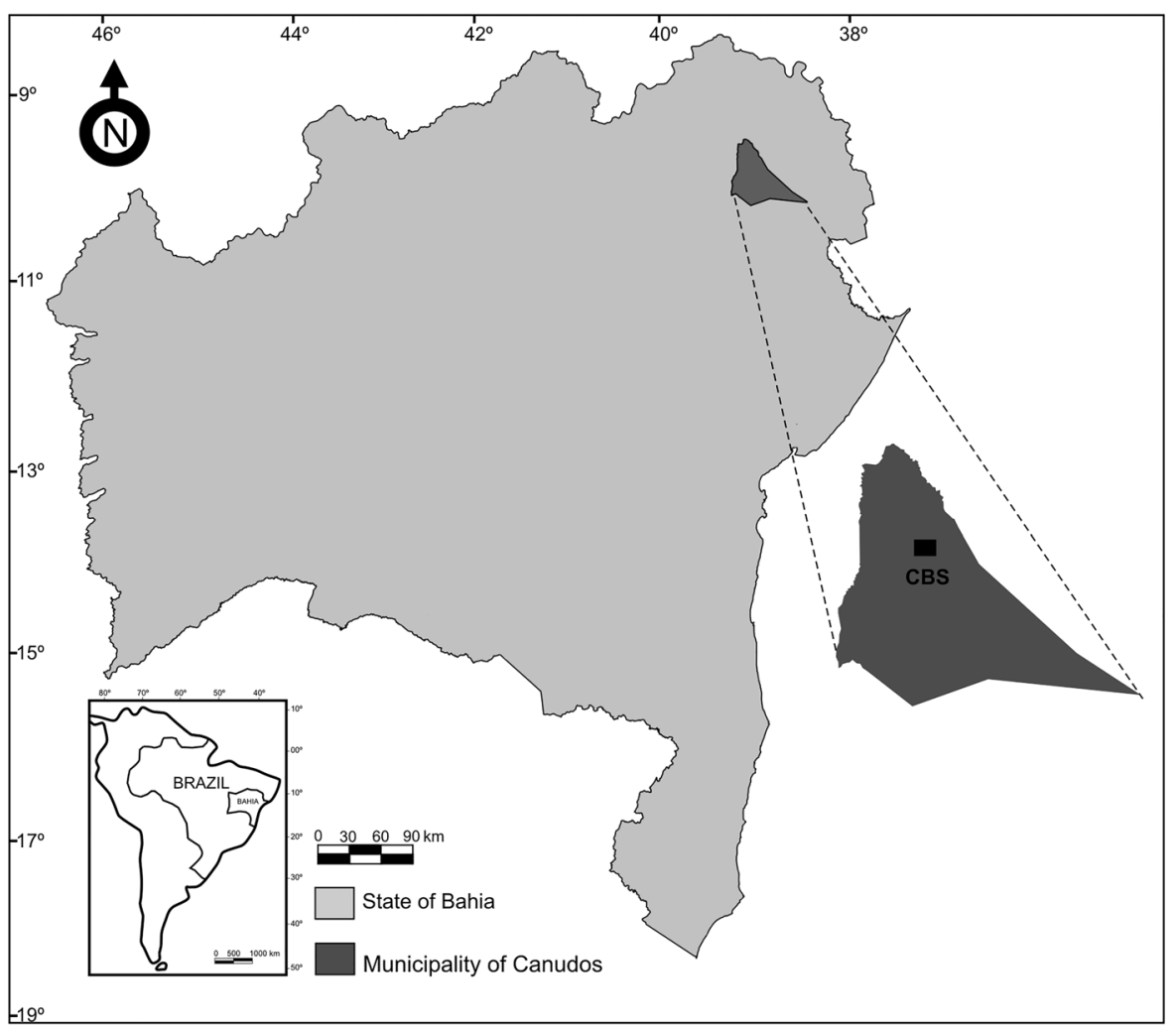

Figure 1. Location of moss polsters in Canudos Biological Station, Bahia State, Semi-arid of Brazil. 


\section{Sample processing}

Samples were processed using the method of Grabandt (1980) with modifications due to the specific conditions of the samples. Thus, the following procedures were performed: (1) $4 \mathrm{~cm}^{2}$ was cut from each sample; (2) the material was disrupted with a glass rod, after which two tablets of exotic spore (Lycopodium clavatum L. spores, ca. 18,583 per tablet, previously dissolved in $3 \mathrm{ml}$ of $10 \% \mathrm{HCl}$ ) were added, and the sample boiled for 20 minutes in $10 \mathrm{ml}$ of $10 \% \mathrm{KOH}$ solution; (3) the mixture was filtered through a steel mesh with an approximately $198-\mu \mathrm{m}$ aperture, and the final liquid was transferred to a polypropylene test tube for centrifugation; (4) the residue was washed with distilled water and centrifuge; (5) the final residue was then incubated in acetic acid for $5 \mathrm{~min}$ and centrifuged again; (6) acetolysis was performed according to Erdtman (1960); and (7) finally, six permanent slides were mounted with glycerin jelly and melted paraffin for each sample. All centrifugation steps were performed at 2,500 rpm.

\section{Qualitative and quantitative analysis}

Whenever possible, pollen grains were identified at the species level, using the pollen catalog available for the local flora (Silva 2007) and other works containing pollen descriptions of genera and families recorded in the CBS. We also consulted the pollen collection of the Laboratory of Palynological Studies, Universidade do Estado da Bahia (UNEB /Campus VII) and the Laboratory of Plant Micromorphology, Universidade Estadual de Feira de Santana (UEFS), which have slides of several species that are found in the Brazilian semiarid region. For the quantitative analy- sis, we sought to count at least 500 pollen grains for each sample. To calculate pollen concentration per $\mathrm{cm}^{2}$, we used a rule of three, wherein the amount of pollen grains for each pollen type is multiplied by the total number of spores of L. clavatum contained in the tablets, and finally divided by the number of spores counted per sample. This result was then divided by four (value in $\mathrm{cm}^{2}$ used for each bryophyte sample) to express the pollen concentration per $\mathrm{cm}^{2}$.

\section{Comparative analysis}

Comparative analysis was performed with the pollen spectra obtained. We performed a cluster analysis, using the Dice similarity coefficient (McCune \& Grace 2002), to evaluate the formation of groups among the samples studied based on the pollen types identified in the samples. All pollen types identified were considered for the presenceabsence data matrix (Hammer et al. 2001). The similarity analysis was performed with the software PAST - Palaeontological Statistics - version 3.07 (Hammer et al. 2001).

\section{Results}

\section{Pollen spectra and representative pollen types}

All of the moss polsters samples on rocks (MPR) and in soil (MPS) contained pollen grains. Of the 372 total types registered, 140 had their taxonomical affinity identified (Tab. 1) and 232 were unidentified. Among the identified pollen types, 58 had a botanical affinity established at the species level, 47 at the genus level, and 35 at the family level.

Table 1. Concentration (pollen grains $/ \mathrm{cm}^{2}$ ) of pollen types found in moss polsters at the Canudos Biological Station, in the state of Bahia, Brazil.

\begin{tabular}{lccccccccc}
\hline \multirow{2}{*}{ Pollen types (related botanic family) } & \multicolumn{3}{c}{$\mathbf{2 0 0 3}$} & \multicolumn{5}{c}{$\mathbf{2 0 0 5}$} \\
\cline { 2 - 7 } & MPR1 & MPR2 & MPS1 & MPR3 & MPR4 & MPR5 & MPS2 & MPS3 & Sum \\
\hline 1. Mitracarpus/Spermacoce (Rubiaceae) & 43.20 & 226.62 & $1,606.94$ & 471.64 & 83.58 & 27.98 & $12,344.78$ & $1,906.60$ & $16,711.34$ \\
2. Conocliniopsis prasiffolia (Asteraceae) & 172.86 & 212.44 & $1,019.78$ & 62.88 & - & 7.98 & $8,607.14$ & $6,101.12$ & $16,184.20$ \\
3. Copaifera (Fab./Caesalpinioideae) & 86.42 & $8,016.74$ & 154.50 & $1,933.76$ & 706.96 & 599.70 & 368.50 & 432.16 & $12,298.74$ \\
4. Trichogonia campestris (Asteraceae) & 8.64 & 14.16 & $1,885.08$ & - & 3.48 & 43.96 & $6,290.84$ & $3,190.36$ & $11,436.52$ \\
5. Pityrocarpa moniliformis (Fab./Mimosoideae) & 276.58 & $4,178.34$ & 164.80 & 31.44 & 114.92 & 299.84 & 263.21 & 101.68 & $5,430.81$ \\
6. Celtis (Cannabaceae) & 43.20 & 56.64 & - & $3,270.10$ & 10.44 & 19.98 & - & - & $3,400.36$ \\
7. Alternanthera ramosissima (Amaranthaceae) & 8.64 & 42.48 & 401.72 & 691.75 & - & - & 658.02 & 419.44 & $2,222.05$ \\
8. Commiphora leptophloeos (Burseraceae) & 8.64 & 325.76 & 432.64 & - & 24.36 & 27.98 & $1,079.18$ & 317.76 & $2,216.32$ \\
9. Trachypogon spicatus (Poaceae) & - & - & - & 15.72 & - & - & $1,210.78$ & 394.02 & $1,620.52$ \\
10. Anadenanthera colubrina (Fab./Mimosoideae) & 8.64 & $1,033.96$ & 30.9 & 47.16 & 16.92 & 79.96 & 50.64 & 63.54 & $1,331.72$ \\
11. Varronia globosa (Boraginaceae) & - & 155.80 & 175.1 & - & 6.96 & 23.98 & 526.42 & 381.32 & $1,269.58$ \\
12. Platypodanthera (Asteraceae) & 484.02 & 368.26 & 247.22 & 47.16 & 3.48 & 7.98 & - & - & $1,158.12$ \\
13. Cereus jamacaru (Cactaceae) & - & 56.64 & 113.30 & - & - & 15.98 & 289.52 & 406.74 & 882.18 \\
14. Acalypha brasiliensis (Euphorbiaceae) & 8.64 & 14.16 & 473.84 & - & - & 15.98 & - & 279.62 & 792.24 \\
\hline
\end{tabular}


Table 1. Continuation.

\begin{tabular}{|c|c|c|c|c|c|c|c|c|c|}
\hline \multirow{2}{*}{ Pollen types (related botanic family) } & \multicolumn{3}{|c|}{2003} & \multicolumn{5}{|c|}{2005} & \multirow{2}{*}{ Sum } \\
\hline & MPR1 & MPR2 & MPS1 & MPR3 & MPR4 & MPR5 & MPS2 & MPS3 & \\
\hline 15. Vernonia (Asteraceae) & - & - & - & - & - & - & 605.38 & 152.52 & 757.90 \\
\hline 16. Piptadenia stipulacea (Fab./Mimosoideae) & 129.64 & 538.22 & - & 31.44 & 6.96 & 7.98 & - & - & 714.24 \\
\hline 17. Stilpnopappus scaposus (Asteraceae) & 207.43 & 467.40 & - & - & 6.96 & 3.98 & - & - & 685.77 \\
\hline 18. Mimosa sensitiva (Fab./Mimosoideae) & 25.92 & 14.16 & 92.70 & 110.04 & 17.40 & 31.98 & 368.50 & - & 660.70 \\
\hline 19. Malpighiaceae 4 & - & - & 103.00 & - & - & - & 500.10 & 25.42 & 628.52 \\
\hline 20. Mimosa arenosa (Fab./Mimosoideae) & - & 396.58 & 164.80 & 31.44 & 10.44 & - & - & - & 603.26 \\
\hline 21. Herissantia (Malvaceae) & 43.20 & 382.42 & 41.20 & 47.16 & 3.48 & 7.98 & 50.64 & 12.70 & 588.78 \\
\hline 22. Chamaecrista swainsonii (Fab./Caesalpinioideae) & 25.92 & 424.90 & - & 47.16 & 20.88 & 19.98 & - & 38.12 & 576.96 \\
\hline 23. Campomanesia (Myrtaceae) & 17.28 & 396.58 & - & 94.32 & 13.93 & 39.98 & - & - & 562.09 \\
\hline 24. Eupatorium (Asteraceae) & - & - & 504.74 & - & - & - & - & - & 504.74 \\
\hline 25. Amaranthus viridis (Amaranthaceae) & - & 42.48 & 82.40 & 62.88 & - & - & 105.28 & 177.94 & 470.98 \\
\hline 26. Mimosa lewisii (Fab./Mimosoideae) & 60.50 & 212.44 & - & 140.00 & 17.40 & 3.98 & - & 12.70 & 447.02 \\
\hline 27. Plathymenia reticulata (Fab./Mimosoideae) & - & 439.08 & - & - & - & - & - & - & 439.08 \\
\hline 28. Zizyphus joazeiro (Rhamnaceae) & 60.50 & 509.88 & - & 15.72 & 3.48 & 31.98 & - & - & 621.56 \\
\hline 29. Enteropogon mollis (Poaceae) & - & - & 391.42 & - & - & - & - & - & 391.42 \\
\hline 30. Chamaecrista ramosa (Fab./Caesalpinioideae) & - & 354.08 & - & - & - & - & - & - & 354.08 \\
\hline 31. Gomphrena demissa (Amaranthaceae) & - & - & 41.20 & - & - & - & 157.92 & 127.10 & 326.22 \\
\hline 32. Pilosocereus tuberculatus (Cactaceae) & - & 297.44 & 20.60 & - & 3.48 & - & - & - & 321.52 \\
\hline 33. Froelichia lanata (Amaranthaceae) & - & - & 113.30 & - & - & - & 131.60 & 63.54 & 308.44 \\
\hline 34. Sida (Malvaceae) & - & 42.48 & 41.20 & 15.72 & - & 3.98 & 26.32 & 165.22 & 294.92 \\
\hline 35. Anemopaegma laeve (Bignoniaceae) & - & - & 51.50 & - & - & - & 210.56 & 25.42 & 287.48 \\
\hline 36. Myrcia 1 (Myrtaceae) & - & 226.62 & - & 15.72 & - & 3.98 & - & - & 246.32 \\
\hline 37. Asteraceae 3 & - & - & 164.80 & - & - & - & - & 76.26 & 241.06 \\
\hline 38. Phyllanthus (Phyllanthaceae) & - & - & 123.60 & 62.88 & 6.96 & 43.96 & - & - & 237.40 \\
\hline 39. Clidemia (Melastomataceae) & - & 212.44 & - & - & - & - & - & - & 212.44 \\
\hline 40. Aspilia bonplandiana (Asteraceae) & 34.57 & - & 154.50 & - & 10.44 & 3.98 & - & - & 203.49 \\
\hline 41. Myrtaceae 3 & - & 184.12 & - & - & 17.40 & - & - & - & 201.52 \\
\hline 42. Chomelia (Rubiaceae) & 8.64 & 184.12 & - & - & 6.96 & - & - & - & 199.72 \\
\hline 43. Croton zehntneri (Euphorbiaceae) & - & 70.81 & 30.90 & 15.72 & 6.96 & 39.98 & - & 12.70 & 177.07 \\
\hline 44. Harpochilus (Acanthaceae) & - & - & 10.30 & 15.72 & 6.96 & - & - & 139.80 & 172.78 \\
\hline 45. Hyptis (Lamiaceae) & 25.92 & 113.30 & 20.60 & - & - & - & - & 12.70 & 172.52 \\
\hline 46. Senna (Fab./Caesalpinioideae) & - & - & 30.90 & - & - & - & 131.60 & - & 162.50 \\
\hline 47. Poaceae & - & - & 154.50 & - & - & - & - & - & 154.50 \\
\hline 48. Jacquemontia 1 (Convolvulaceae) & 17.28 & - & 82.40 & - & - & - & 50.64 & - & 150.32 \\
\hline 49. Solanum megalonyx (Solanaceae) & 8.64 & 42.48 & - & 62.88 & 27.86 & 3.98 & - & - & 145.84 \\
\hline 50. Erythroxylum caatingae (Erythroxylaceae) & - & 127.46 & - & - & - & 15.96 & - & - & 143.42 \\
\hline 51. Melocactus zehntneri (Cactaceae) & - & 42.48 & - & - & - & 7.98 & 50.64 & 38.12 & 139.22 \\
\hline 52. Croton heliotropiifolius (Euphorbiaceae) & - & 28.32 & - & 62.88 & 27.86 & 7.98 & - & - & 127.04 \\
\hline 53. Jatropha ribifolia (Euphorbiaceae) & - & 99.14 & - & - & 6.96 & 19.98 & - & - & 126.08 \\
\hline 54. Bignoniaceae & - & - & 92.70 & 31.44 & - & - & - & - & 124.14 \\
\hline 55. Asteraceae 2 & - & - & 123.60 & - & - & - & - & - & 123.60 \\
\hline 56. Digitaria tenuis (Poaceae) & - & 28.32 & 61.80 & - & - & 3.98 & 26.32 & - & 120.42 \\
\hline 57. Helicteres (Malvaceae) & - & - & - & 94.32 & - & - & - & - & 94.32 \\
\hline 58. Rubiaceae 1 & - & - & - & 94.32 & - & - & - & - & 94.32 \\
\hline
\end{tabular}


Table 1. Continuation.

\begin{tabular}{|c|c|c|c|c|c|c|c|c|c|}
\hline \multirow{2}{*}{ Pollen types (related botanic family) } & \multicolumn{3}{|c|}{2003} & \multicolumn{5}{|c|}{2005} & \multirow{2}{*}{ Sum } \\
\hline & MPR1 & MPR2 & MPS1 & MPR3 & MPR4 & MPR5 & MPS2 & MPS3 & \\
\hline 59. Myrtaceae 2 & - & 84.98 & - & - & 3.48 & - & - & - & 88.46 \\
\hline 60. Poincianella pyramidalis (Fab./Caesalpinioideae) & 17.28 & 70.81 & - & - & - & - & - & - & 88.09 \\
\hline 61. Andropogon selloanus (Poaceae) & 17.28 & - & 10.30 & - & - & 7.98 & 26.32 & 25.42 & 87.30 \\
\hline 62. Solanaceae 1 & - & - & - & - & - & 7.98 & - & 76.26 & 84.24 \\
\hline 63. Heteropterys (Malpighiaceae) & - & - & 30.90 & - & - & - & 50.64 & - & 81.54 \\
\hline 64. Rubiaceae 2 & - & - & - & 78.60 & - & - & - & - & 78.60 \\
\hline 65. Myrtaceae 4 & - & 70.81 & - & - & - & - & - & - & 70.81 \\
\hline 66. Eucalyptus (Myrtaceae) & - & 42.48 & - & - & - & - & 26.32 & - & 68.80 \\
\hline 67. Cnidoscolus loefgrenii (Euphorbiaceae) & 8.64 & 56.64 & - & - & - & - & - & - & 65.28 \\
\hline 68. Verbenaceae & - & - & - & - & - & - & - & 63.54 & 63.54 \\
\hline 69. Barnebya harleyi (Malpighiaceae) & - & - & - & 47.16 & 3.48 & - & - & 12.70 & 63.34 \\
\hline 70. Mimosa misera (Fab./Mimosoideae) & - & 42.48 & 20.60 & - & - & - & - & - & 63.08 \\
\hline 71. Diodia (Rubiaceae) & 8.64 & 28.32 & - & - & - & 11.99 & - & 12.70 & 61.65 \\
\hline 72. Malpighiaceae 2 & - & - & 10.30 & - & - & - & 50.64 & - & 60.94 \\
\hline 73. Spondias tuberosa (Anacardiaceae) & - & - & - & - & 24.36 & 7.98 & 26.32 & - & 58.66 \\
\hline 74. Jatropha mutabilis (Euphorbiaceae) & - & 56.64 & - & - & - & - & - & - & 56.64 \\
\hline 75. Mimosa 2 (Fab./Mimosoideae) & - & - & - & - & - & 27.98 & 26.32 & - & 54.30 \\
\hline 76. Banisteriopsis stellaris (Malpighiaceae) & - & - & 10.30 & 15.72 & - & - & 26.32 & - & 52.34 \\
\hline 77. Melochia (Malvaceae) & - & 28.32 & - & - & 17.40 & - & - & - & 45.72 \\
\hline 78. Mimosa 1 (Fab./Mimosoideae) & - & - & - & - & 45.26 & - & - & - & 45.26 \\
\hline 79. Piriadacus erubescens (Bignoniaceae) & - & - & - & - & 6.96 & - & - & 38.12 & 45.08 \\
\hline 80. Jacquemontia montana (Convolvulaceae) & - & 42.48 & - & - & - & - & - & - & 42.48 \\
\hline 81. Tacinga inamoema (Cactaceae) & - & 28.32 & - & - & - & - & - & 12.70 & 41.02 \\
\hline 82. Fabaceae/Mimosoideae & - & 14.16 & - & - & - & - & 26.32 & - & 40.48 \\
\hline 83. Oxalis psoralioides (Oxalidaceae) & - & 28.32 & - & - & 3.48 & 7.98 & - & - & 39.78 \\
\hline 84. Rutaceae & 34.57 & - & - & - & - & 3.98 & - & - & 38.55 \\
\hline 85. Lepidaploa aurea (Asteraceae) & 25.92 & - & 10.30 & - & - & - & - & - & 36.22 \\
\hline 86. Polygalaceae & - & - & - & - & - & 7.98 & - & 25.42 & 33.40 \\
\hline 87. Cardiospermum corindum (Sapindaceae) & 8.64 & - & - & - & 13.92 & - & - & 12.70 & 35.26 \\
\hline 88. Cyperaceae & - & - & - & 31.44 & - & - & - & - & 31.44 \\
\hline 89. Byrsonima (Malpighiaceae) & - & - & 30.90 & - & - & - & - & - & 30.90 \\
\hline 90. Malpighiaceae 1 & - & - & 30.90 & - & - & - & - & - & 30.90 \\
\hline 91. Cleome (Capparaceae) & 17.28 & - & - & - & 3.48 & 7.98 & - & - & 28.74 \\
\hline 92. Lamiaceae & - & 42.48 & 20.60 & - & - & 7.98 & - & - & 71.06 \\
\hline 93. Loranthaceae 1 & - & 28.32 & - & - & - & - & - & - & 28.32 \\
\hline 94. Poeppigia procera (Fab./Caesalpinioideae) & - & 28.32 & - & - & - & - & - & - & 28.32 \\
\hline 95. Roupala (Proteaceae) & - & 28.32 & - & - & - & - & - & - & 28.32 \\
\hline 96. Solanaceae 3 & - & 14.16 & - & - & - & - & - & 12.70 & 26.86 \\
\hline 97. Myrcia 3 (Myrtaceae) & - & - & - & - & - & - & 26.32 & - & 26.32 \\
\hline 98. Malpighiaceae 3 & - & 14.16 & 10.30 & - & - & - & - & - & 24.46 \\
\hline 99. Myrcia 2 (Myrtaceae) & - & - & - & - & 3.48 & 19.98 & - & - & 23.46 \\
\hline 100. Manihot dichotoma (Euphorbiaceae) & - & 14.16 & - & - & 6.96 & - & - & - & 21.12 \\
\hline
\end{tabular}


Table 1. Continuation.

\begin{tabular}{|c|c|c|c|c|c|c|c|c|c|}
\hline \multirow{2}{*}{ Pollen types (related botanic family) } & \multicolumn{3}{|c|}{2003} & \multicolumn{5}{|c|}{2005} & \multirow{2}{*}{ Sum } \\
\hline & MPR1 & MPR2 & MPS1 & MPR3 & MPR4 & MPR5 & MPS2 & MPS3 & \\
\hline 101. Malvaceae & - & - & 20.60 & - & - & - & - & - & 20.60 \\
\hline 102. Vernonia chalybaea (Asteraceae) & - & - & - & 15.72 & - & 3.98 & - & - & 19.70 \\
\hline 103. Poincianella microphylla (Fab./Caesalpinioideae) & - & 14.16 & - & - & - & 3.98 & - & - & 18.14 \\
\hline 104. Angelonia (Scrophulariaceae) & 17.28 & - & - & - & - & - & - & - & 17.28 \\
\hline 105. Senna rizzinii (Fab./Caesalpinioideae) & 17.28 & - & - & - & - & - & - & - & 17.28 \\
\hline 106. Solanum paniculatum (Solanaceae) & 17.28 & - & - & - & - & - & - & - & 17.28 \\
\hline 107. Jacquemontia 2 (Convolvulaceae) & - & - & - & - & 3.48 & - & - & 12.70 & 16.18 \\
\hline 108. Fabaceae & - & - & - & - & - & 15.98 & - & - & 15.98 \\
\hline 109. Chamaecrista (Fab./Caesalpinioideae) & - & 14.16 & - & - & - & - & - & - & 14.16 \\
\hline 110. Loranthaceae 2 & - & 14.16 & - & - & - & - & - & - & 14.16 \\
\hline 111. Paullinia (Sapindaceae) & - & 14.16 & - & - & - & - & - & - & 14.16 \\
\hline 112. Psittacanthus (Loranthaceae) & - & 14.16 & - & - & - & - & - & - & 14.16 \\
\hline 113. Ipomoea (Convolvulaceae) & - & - & - & - & - & - & - & 12.70 & 12.70 \\
\hline 114. Solanum (Solanaceae) & - & - & - & - & - & - & - & 12.70 & 12.70 \\
\hline 115. Zornia (Fab./Papilionoideae) & 8.64 & - & - & - & - & 3.98 & - & - & 12.62 \\
\hline 116. Microtea (Phytolaccaceae) & 8.64 & - & - & - & 3.48 & - & - & - & 12.12 \\
\hline 117. Simaba (Simaroubaceae) & - & - & - & - & - & 11.99 & - & - & 11.99 \\
\hline 118. Mollugo verticillata (Molluginaceae) & - & - & - & - & 6.96 & 3.98 & - & - & 10.94 \\
\hline 119. Euphorbiaceae & - & - & - & - & 10.44 & - & - & - & 10.44 \\
\hline 120. Myrtaceae 1 & - & - & - & - & 10.44 & - & - & - & 10.44 \\
\hline 121. Senna velutina (Fab./Caesalpinioideae) & - & - & - & - & 10.44 & - & - & - & 10.44 \\
\hline 122. Asteraceae 1 & - & - & 10.30 & - & - & - & - & - & 10.30 \\
\hline 123. Polygala (Polygalaceae) & - & - & 10.30 & - & - & - & - & - & 10.30 \\
\hline 124. Solanaceae 2 & - & - & 10.30 & - & - & - & - & - & 10.30 \\
\hline 125. Anacardiaceae & 8.64 & - & - & - & - & - & - & - & 8.64 \\
\hline 126. Capparaceae & 8.64 & - & - & - & - & - & - & - & 8.64 \\
\hline 127. Froelichia humboldtiana (Amaranthaceae) & 8.64 & - & - & - & - & - & - & - & 8.64 \\
\hline 128. Miconia (Melastomataceae) & 8.64 & - & - & - & - & - & - & - & 8.64 \\
\hline 129. Passifloraceae & 8.64 & - & - & - & - & - & - & - & 8.64 \\
\hline 130. Simaroubaceae & 8.64 & - & - & - & - & - & - & - & 8.64 \\
\hline 131. Waltheria (Malvaceae) & 8.64 & - & - & - & - & - & - & - & 8.64 \\
\hline 132. Ouratea (Ochnaceae) & - & - & - & - & - & 7.98 & - & - & 7.98 \\
\hline 133. Jatropha (Euphorbiaceae) & - & - & - & - & 3.48 & 3.98 & - & - & 7.46 \\
\hline 134. Apocynaceae & - & - & - & - & - & 3.98 & - & - & 3.98 \\
\hline 135. Aspicarpa harleyi (Malpighiaceae) & - & - & - & - & - & 3.98 & - & - & 3.98 \\
\hline 136. Acanthaceae & - & - & - & - & 3.48 & 3.98 & - & - & 7.46 \\
\hline 137. Anacardium occidentale (Anacardiaceae) & - & - & - & - & 3.48 & - & - & - & 3.48 \\
\hline 138. Heisteria (Olacaceae) & - & - & - & - & 3.48 & - & - & - & 3.48 \\
\hline 139. Securidaca (Polygalaceae) & - & - & - & - & 3.48 & - & - & - & 3.48 \\
\hline 140. Simarouba (Simaroubaceae) & - & - & - & - & 3.48 & - & - & - & 3.48 \\
\hline $\begin{array}{c}\text { [Number of unidentified pollen types] } \\
\text { Pollen grains } / \mathrm{cm}^{2}\end{array}$ & $\begin{array}{c}{[14]} \\
155.52\end{array}$ & $\begin{array}{c}{[75]} \\
3,441.32\end{array}$ & $\begin{array}{c}{[56]} \\
1,792.24\end{array}$ & $\begin{array}{c}{[19]} \\
628.80\end{array}$ & $\begin{array}{c}{[30]} \\
177.48\end{array}$ & $\begin{array}{c}{[56]} \\
378.93\end{array}$ & $\begin{array}{c}{[15]} \\
1,338.34\end{array}$ & $\begin{array}{c}{[18]} \\
559.14\end{array}$ & - \\
\hline $\begin{array}{c}\text { [Number of identified pollen types] } \\
\text { Pollen grains } / \mathrm{cm}^{2}\end{array}$ & $\begin{array}{c}{[44]} \\
2,082.77\end{array}$ & $\begin{array}{c}{[64]} \\
21,415.23\end{array}$ & $\begin{array}{c}{[50]} \\
9,641.37\end{array}$ & $\begin{array}{c}{[33]} \\
7,812.01\end{array}$ & $\begin{array}{c}{[51]} \\
1,392.25\end{array}$ & $\begin{array}{c}{[53]} \\
1,662.46\end{array}$ & $\begin{array}{c}{[33]} \\
34,390.05\end{array}$ & $\begin{array}{c}{[40]} \\
15,404.98\end{array}$ & - \\
\hline Total number of pollen grains $/ \mathrm{cm}^{2}$ & $2,238.29$ & $24,856.55$ & $11,433.62$ & $8,440.81$ & $1,569.73$ & $2,041.39$ & $35,728.39$ & $15,964.12$ & - \\
\hline
\end{tabular}


The failure to identify the undetermined types was mainly due to the pollen being crumpled or deformed or in an unfavorable position for observing important features of the exine, such as details of ornamentation and openings.

The families with the highest representation from the pollen types were Fabaceae $(\mathrm{n}=23)$ and Asteraceae $(\mathrm{n}=12)$, followed by Euphorbiaceae, Malpighiaceae and Myrtaceae $($ all $n=9)$. The pollen types identified only at the genus level had 39 records of respective genera in CBS, and there was no local record for eight types [Celtis (Cannabaceae), Eucalyptus (Myrtaceae), Heisteria (Olacaceae), Miconia (Melastomataceae), Paullinia (Sapindaceae), Roupala (Proteaceae), Securidaca (Polygalaceae) and Simarouba (Simaroubaceae)]. Among the 58 pollen types identified at the species level, 54 were related to species that were recorded in the flora of CBS, while the records were restricted to the genus level for the other four pollen types [Banisteriopsis stellaris (Malpighiaceae), Froelichia lanata (Amaranthaceae), Mimosa arenosa (Fabaceae/Mimosoideae) and Senna velutina (Fabaceae/ Caesalpinioideae)].
Despite a qualitative difference, the pollen concentrations/ $\mathrm{cm}^{2}$ were very similar between 2003 and $2005(12,842.82$ and $12,748.88$ pollen grains $/ \mathrm{cm}^{2}$, respectively). As for the two types of substrates in which the moss polsters were collected, the soil samples contained higher pollen concentrations $(21,042.04$ pollen grains $\left./ \mathrm{cm}^{2}\right)$ than the rock samples $(7,829.35$ pollen grains $\left./ \mathrm{cm}^{2}\right)$. On the other hand, $68(48.57 \%)$ pollen types were unique to the rock samples, whereas only 24 were unique to the soil samples (17.14\%). Forty-eight other pollen types (34.28\%) had records in both the rock and soil samples (Tab. 1).

Among the pollen types, only five were recorded in all samples from the two years [Mitracarpus/Spermacoce (Rubiaceae), Copaifera (Fabaceae/Caesalpinioideae), Pityrocarpa moniliformis (Fabaceae/Mimosoideae), Anadenanthera colubrina (Fabaceae/Mimosoideae), and Herissantia (Malvaceae)]. Moreover, the sums of the concentrations of these pollen types varied substantially, from 16,711.34 pollen grains of Mitracarpus/Spermacoce $(1,876.76$ in 2003 and $14,834.58$ in 2005) to 588.78 pollen grains of Herissantia (Tab. 1).

Table 2. Growth habits of taxa related to the pollen types found in moss polsters at the Canudos Biological Station, in the state of Bahia, Brazil.

\begin{tabular}{|c|c|}
\hline $\begin{array}{l}\text { Habits* }^{*} \\
\%\end{array}$ & Pollen types \\
\hline $\begin{array}{c}\text { Shrub } \\
26.43 \%\end{array}$ & $\begin{array}{l}\text { Acalyphabrasiliensis(Euphorbiaceae), Acanthaceae,Aspicarpaharleyi(Malpighiaceae), Byrsonima(Malpighiaceae), Campomanesia(Myrtaceae), } \\
\text { Chomelia (Rubiaceae), Clidemia (Melastomataceae), Cnidoscolus loefgrenii (Euphorbiaceae), Croton heliotropiifolius (Euphorbiaceae), Croton } \\
\text { zehntneri (Euphorbiaceae), Eupatorium (Asteraceae), Harpochilus (Acanthaceae), Heisteria (Olacaceae), Helicteres (Malvaceae), Heteropterys } \\
\text { (Malpighiaceae), Jatropha (Euphorbiaceae), Jatropha mutabilis (Euphorbiaceae), Jatropha ribifolia (Euphorbiaceae), Manihot dichotoma } \\
\text { (Euphorbiaceae), Melochia (Malvaceae), Mimosa arenosa (Fabaceae/Mimosoideae), Mimosa lewisii (Fabaceae/Mimosoideae), Myrcia } 1 \\
\text { (Myrtaceae), Myrcia } 2 \text { (Myrtaceae), Myrcia } 3 \text { (Myrtaceae), Myrtaceae 1, Myrtaceae 2, Myrtaceae 3, Myrtaceae 4, Securidaca (Polygalaceae), } \\
\text { Senna (Fabaceae/Caesalpinioideae), Senna velutina (Fabaceae/Caesalpinioideae), Solanaceae 2, Solanum (Solanaceae), Solanum megalonyx } \\
\text { (Solanaceae), Solanum paniculatum (Solanaceae), Varronia globosa (Boraginaceae). }\end{array}$ \\
\hline $\begin{array}{l}\text { Herb } \\
22.14 \%\end{array}$ & $\begin{array}{l}\text { Alternanthera ramosissima (Amaranthaceae), Amaranthus viridis (Amaranthaceae), Andropogon selloanus (Poaceae), Angelonia } \\
\text { (Scrophulariaceae), Aspilia bonplandiana (Asteraceae), Cardiospermum corindum (Sapindaceae), Cleome (Capparaceae), Conocliniopsis } \\
\text { prasiifolia (Asteraceae), Cyperaceae, Digitaria tenuis (Poaceae), Diodia (Rubiaceae), Enteropogon mollis (Poaceae), Froelichia humboldtiana } \\
\text { (Amaranthaceae), Froelichia procera (Amaranthaceae), Gomphrena demissa (Amaranthaceae), Microtea (Phytolaccaceae), Mimosa } 2 \text { (Fabaceae/ } \\
\text { Mimosoideae), Mitracarpus/Spermacoce (Rubiaceae), Mollugo verticillata (Molluginaceae), Oxalis psoralioides (Oxalidaceae), Phyllanthus } \\
\text { (Phyllanthaceae), Platypodanthera (Asteraceae), Poaceae, Polygala (Polygalaceae), Polygalaceae, Sida (Malvaceae), Stilpnopappus scaposus } \\
\text { (Asteraceae), Trachypogon spicatus (Poaceae), Trichogonia campestris (Asteraceae), Vernonia (Asteraceae), Zornia (Fabaceae/Papilionoideae). }\end{array}$ \\
\hline $\begin{array}{c}\text { Tree } \\
18.57 \%\end{array}$ & $\begin{array}{l}\text { Anacardiaceae, Anacardium occidentale (Anacardiaceae), Anadenanthera colubrina (Fabaceae/Mimosoideae), Barnebya harleyi } \\
\text { (Malpighiaceae), Celtis (Ulmaceae), Commiphora leptophloeos (Burseraceae), Copaifera (Fabaceae/Caesalpinioideae), Erythroxylum caatingae } \\
\text { (Erythroxylaceae), Eucalyptus (Myrtaceae), Miconia (Melastomataceae), Ouratea (Ochnaceae), Piptadenia stipulacea (Fabaceae/Mimosoideae), } \\
\text { Piriadacus erubescens (Bignoniaceae), Pityrocarpa moniliformis (Fabaceae/Mimosoideae), Plathymenia reticulata (Fabaceae/Mimosoideae), } \\
\text { Poeppigia procera (Fabaceae/Caesalpinioideae), Poincianella microphylla (Fabaceae/Caesalpinioideae), Poincianella pyramidalis (Fabaceae/ } \\
\text { Caesalpinioideae), Roupala (Proteaceae), Rutaceae, Senna rizzinii (Fabaceae/Caesalpinioideae), Simaba (Simaroubaceae), Simarouba } \\
\text { (Simaroubaceae), Simaroubaceae, Spondias tuberosa (Anacardiaceae), Zizyphus joazeiro (Rhamnaceae). }\end{array}$ \\
\hline $\begin{array}{l}\text { Subshrub } \\
8.57 \%\end{array}$ & $\begin{array}{l}\text { Chamaecrista (Fabaceae/Caesalpinioideae), Chamaecrista ramosa (Fabaceae/Caesalpinioideae), Chamaecrista swainsonii (Fabaceae/ } \\
\text { Caesalpinioideae), Herissantia (Malvaceae), Hyptis (Lamiaceae), Lamiaceae, Lepidaploa aurea (Asteraceae), Mimosa } 1 \text { (Fabaceae/Mimosoideae), } \\
\text { Mimosa misera (Fabaceae/Mimosoideae), Mimosa sensitiva (Fabaceae/Mimosoideae), Vernonia muricata (Asteraceae), Waltheria (Malvaceae). }\end{array}$ \\
\hline $\begin{array}{l}\text { Liana } \\
6.43 \%\end{array}$ & $\begin{array}{l}\text { Anemopaegma laeve (Bignoniaceae), Apocynaceae, Banisteriopsis stellaris (Malpighiaceae), Ipomoea (Convolvulaceae), Jacquemontia } 1 \\
\text { (Convolvulaceae), Jacquemontia } 2 \text { (Convolvulaceae), Jacquemontia montana (Convolvulaceae), Passifloraceae, Paullinia (Sapindaceae). }\end{array}$ \\
\hline $\begin{array}{r}\text { Cacti } \\
2.86 \%\end{array}$ & Cereus jamacaru (Cactaceae), Melocactus zehntneri (Cactaceae), Pilosocereus tuberculatus (Cactaceae), Tacinga inamoema (Cactaceae). \\
\hline $\begin{array}{l}\text { Undefined } \\
12.86 \%\end{array}$ & $\begin{array}{l}\text { Asteraceae 1, Asteraceae 2, Asteraceae 3, Bignoniaceae, Capparaceae, Euphorbiaceae, Fabaceae, Fabaceae/Mimosoideae, Malpighiaceae 1, } \\
\text { Malpighiaceae 2, Malpighiaceae 3, Malpighiaceae 4, Malvaceae, Rubiaceae 1, Rubiaceae 2, Solanaceae 1, Solanaceae 3, Verbenaceae. }\end{array}$ \\
\hline
\end{tabular}

* According Silva (2007) and Moro et al. (2014) 


\section{Pollen groups based on the cluster analysis}

The resulting phenogram (cophenetic coefficient 0.88 , Fig. 2) presents two groups: rock samples (A) and soil samples (B). The similarity between A and B was only 0.38 . Pollen types related to Fabaceae were predominant in the rock samples, and pollen types related to Asteraceae were predominant in the soil samples. The pollen types most common to both groups and with the highest concentrations $(>5,000.00$ pollen grains $/ \mathrm{cm}^{2}$ ) were Mitracarpus/Spermacoce (Rubiaceae), Conocliniopsis prasiifolia (Asteraceae), Copaifera (Fabaceae/ Caesalpinioideae), Trichogonia campestris (Asteraceae) and Pityrocarpa moniliformis (Fabaceae/Mimosoideae).

The level of similarity within group B (0.54) was higher than within group A (0.46). Specifically, other pollen types also exhibited high concentrations within group $B$ [Alternanthera ramosissima (Amaranthaceae), Eupatorium (Asteraceae), Commiphora leptophloeos (Burseraceae); Acalypha brasiliensis (Euphorbiaceae) and Trachypogon spicatus (Poaceae)] and group A [Platypodanthera, Stilpnopappus scaposus (Asteraceae); Commiphora (Burseraceae); Chamaecrista swainsonii (Fabaceae/Caesalpinioideae); Anadenanthera colubrina, Piptadenia stipulacea, Mimosa lewisii (Fabaceae/Mimosoideae); Campomanesia (Myrtaceae); Zizyphus joazeiro (Rhamnaceae); and Celtis (Cannabaceae)].

The cluster analysis revealed the formation of subgroups within the main groups. Subgroup B1 (MPS1 and MPS2, 2003 and 2005, respectively) had the highest similarity among all groups $(0.62)$. Within group A, subgroup A3 (MPR4 and MPR5, both 2005) showed the greatest similarity (0.58). The other two subgroups, A2 and A1, were formed by samples from 2003 and 2005, respectively, and the similarities for the subgroups were comparable $(0.53$ and 0.48 , respectively).

\section{Representations of life forms and pollination syndromes}

The qualitative analysis showed that the shrub and subshrub habits were most representative of the pollen types related to the CBS species (35.0\%), followed by the herbaceous habit (22.14\%), trees (18.57\%) and other types (cactus, lianas, hemiparasites and indefinite) that together accounted for $24.29 \%$ (Tab. 2).

Through the analysis of the diversity of pollen types represented in the CBS flora (Silva 2007), pollen grains related to taxa with zoophilous pollination were predominant (91.4\%) in the pollen spectrum of moss polsters. On the contrary, pollen grains of anemophilous pollination were represented by only 12 pollen types related to five families [Amaranthaceae: (1) Alternanthera ramosissima, (2) Froelichia humboldtiana, (3) Froelichia lanata, and (4) Gomphrena demissa; (5) Cyperaceae; Erythroxylaceae: (6) Erythroxylum caatingae; Poaceae: (7) Andropogon selloanus, (8) Digitaria tenuis, (9) Enteropogon mollis, (10) Poaceae, and (11) Trachypogon spicatus; and Cannabaceae: (12) Celtis].

\section{Discussion}

Throughout the analysis, the high number of pollen types was surprising considering that the samples came from a semiarid area that generally does not have ideal conditions for the preservation of palinomorphs. The climatic conditions of the CBS may have contributed to the successful representation of pollen types in natural substrates. Considering the predominance of high temperatures, low humidity and high winds for most of the year, the release and deposition of pollen grains are favored (Koff 2001; Veriankaite et al. 2010).

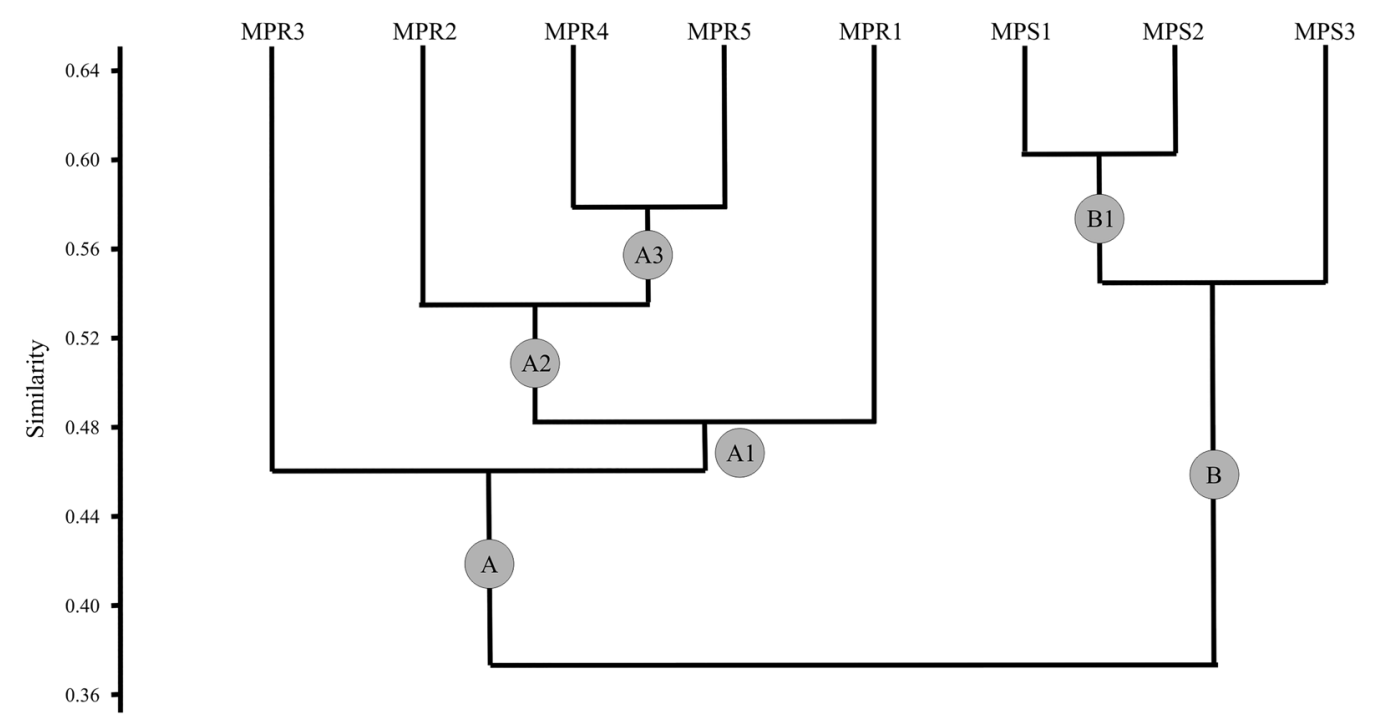

Figure 2. Phenogram generated from de cluster analysis, using the Dice similarity coefficient, of the pollen spectra of eigth samples of moss polsters in Canudos Biological Station, Bahia State, Semi-arid of Brazil. Cophenetic coefficient: 0.88. 
The diversity in the present study was even higher than that reported by Gomes et al. (2014), which is the only available study of natural deposits of pollen grains in the semiarid region of Brazil; the authors recorded 149 pollen types ( 88 determined and 61 indeterminate) in bromeliad tanks in the CBS (Canudos, Bahia). However, unlike these tanks, which were in open areas and in bright sunshine, the moss polsters in the present study were in sheltered areas that were wetter and less exposed to adverse conditions. Thus, one of the reasons for the greater diversity of pollen types in the moss polsters compared to bromeliad tanks may be that the environmental conditions where the samples were collected were more conducive to pollinic preservation.

As mentioned earlier, the two families with the greatest diversity of pollen types were Fabaceae and Asteraceae. In Gomes et al. (2014), these two families, along with Euphorbiaceae, were the most represented in the tanks of bromeliads; the authors also highlighted the diversity of pollen types. According to Forzza et al. (2010), these three families are among the four most diverse families in the domain of the Caatinga. Thus, at this level, the diversity of the pollen types in moss polsters coincided largely with the diversity reported in the Caatinga vegetation. This similarity was also noted by Gomes et al. (2014).

Other studies on pollen rain have been conducted in other biomes with open vegetation in Brazil. Four studies were performed in Rio Grande do Sul State, which is dominated by Campos Sulinos. However, three of the studies were conducted in urban areas with high levels of human disturbance (Bauermann et al. 1998; Bauermann \& Neves 1999; Ávila \& Bauermann 2001), and the other restricted the analysis to pollen types of herbaceous plants (Vergamini et al. 2006). Because these studies did not reflect the natural aspects of vegetation, the results were not considered for comparison in this study.

A fifth study was conducted in the municipality of Aparecida, in Goiás State, an area that is dominated by Cerrado vegetation (Salgado-Labouriau 1973). The author's ranking of families with the highest quantities of pollen grains $/ \mathrm{mg}$ of pollen rain included Poaceae, Fabaceae (Stryphnodendron, Lupinus), Asteraceae (Heliantheae group), Anacardiaceae (Anacardium), Euphorbiaceae (Acalypha, Alchornea), Sapindaceae and Malvaceae (Pseudobombax). Poaceae pollen grains amounted to $73.8 \%$ of the total pollen grains found in the samples. Thus, the most remarkable difference between the pollen spectra of the Caatinga area in the CBS and the Cerrado area in Salgado-Labouriau's study was that eudicots were predominant in the former [(Mitracarpus/ Spermacoce (Rubiaceae), Conocliniopsis prasiifolia (Asteraceae), Copaifera (Fabaceae/Caesalpinioideae), Trichogonia campestris (Asteraceae) and Pityrocarpa moniliformis (Fabaceae/Mimosoideae)], whereas monocots (Poaceae) were predominant in the latter.

In studies of local pollen rain, most of the pollen types are expected to be autochthonous, or related to local spe- cies. This was the case in our study: only four pollen types identified at the species level (Banisteriopsis stellaris, Froelichia lanata, Mimosa arenosa and Senna velutina) and eight identified at the genus level (Celtis, Eucalyptus, Heisteria, Miconia, Paullinia, Roupala, Securidaca and Simarouba) lacked records of the respective taxa in the CBS. Although these taxa are not included in the floristic list for the CBS, the possibility that one or more of them occurs in the area cannot be excluded, as the botanical collections did not cover the entire extent of the area.

The Celtis pollen type, for example, had a high representation in the local pollen rain, with more than 3,400 pollen grains $/ \mathrm{cm}^{2}$. Celtis ranked among the ten pollen types with the highest concentrations (Tab. 1). This bears strong evidence of the possibility of the genus occurring in the CBS without being sampled in the floristic inventory. On the contrary, the possibility that the pollen grains related to other pollen types are from plants from other areas of the region (i.e., allochthonous) should also not be ruled out. Overall, pollen grains from other areas, especially if they were not from anemophilous species, had low representation in the local pollen spectra. For example, the concentrations of other pollen types in the above group were less than 70 pollen grains $/ \mathrm{cm}^{2}$. Moro et al. (2014) recently reported the occurrence of species and genera related to the group mentioned in other areas of Caatinga, with the exception of Roupala.

These pollen types are all possibly related to allochthonous species and insect pollination. An alternative explanation other than long-range wind transport could account for their presence in moss polsters: during the most critical periods of aridity, insect fauna find an alternative source of moisture in moss polsters because of their recognized ability to retain water. This was observed in the CBS during the collections. Every time we accessed the sampling area, Apis mellifera Linnaeus 1758 bees were observed on the moss polsters, mainly along rock walls. The pollen in the honey of these bees is considered contamination, as the pollen may fall from their bodies onto the comb (Jones \& Bryant 1996). This may also occur on the moss polsters. Information in the literature shows that the species A. mellifera $\mathrm{L}$. 1758 has a great capacity for adaptation and exploration in diverse environments, including over several climatic and vegetation zones (Koppler et al. 2007). The bee is considered a generalist because its foraging behavior shows no food preference for specific taxa of plants and can survive on a range of available flora.

The representation of flora in the pollen spectra was more diverse in the rock samples than in the soil samples. Due to the nature of the sandy soil, many pollen grains may have been translocated to the lower levels below the cover of the moss polsters. Additionally, the longevity of the moss polsters needs to be considered. It is probable that the older the moss polsters, the higher the recorded pollen accumulation. The age of the moss polsters was not 
possible to determine, but the moss polsters along the rocky walls appeared to be more developed than those seen in soils. The differing appearance may be a result of the rocky walls, which are constantly wet due to the natural capacity of the sandstone matrix to retain water; therefore, the rocky walls provide ideal conditions for moss polsters to develop and survive for much longer than those in the sandy soil.

Favorable conditions for preserving pollen grains in the moss polsters in the CBS and the possibility of identifying them at the genus or species level were critical for other aspects besides revealing the floristic details. For example, the available information on the habits of the species related to the pollen types (Silva 2007; Moro et al. 2014) allowed the study to verify that $35 \%$ of the pollen types represented shrubs or subshrubs. This percentage as well as the percentages of other types of habits generally corresponded to what was observed in the physiognomy of the local vegetation. Similar results were obtained by Gomes et al. (2014). Furthermore, regarding the pollen representation of local and regional flora in natural collectors, Reese \& Liu (2005) provided an extensive report for Puna vegetation in the central Andes. In a modern pollen rain study of 40 soil surface samples, the authors detected four distinct pollen assemblages in which the pollen types reflected their local vegetation.

Indirectly, aspects related to pollination syndromes in species present in the CBS were also revealed by matching the pollen information with previous data available for the area (Silva 2007). As reported in the results, the predominance of pollen types related to zoophilous species was remarkable. Together, they accounted for $91.4 \%$ of the pollen spectrum of moss polsters. Overall, this representation aligned with expectations, as there is a clear predominance of zoophilous species in the CBS. In addition, as previously mentioned, pollen grains that adhered to the bees that visited the moss polsters probably fell on them. Further evidence of this hypothesis is revealed by the presence of large pollen grains that would not likely have depended on wind for transport. This category of large pollen grains includes those with respective pollen types related to the Cactaceae and Convolvulaceae families, most Euphorbiaceae, many Fabaceae and some Malvaceae. Bawa (1990) analyzed the zoophilous pollination syndromes and found that the insect pollination system was the most common, occurring regardless of the vegetation type.

On the contrary, the low representation of pollen types related to anemophilous species may be linked to the flowering period of the respective species. In the CBS, for example, the five pollen types of grasses that were detected in this study bloom during or immediately after the rainy period. Consequently, rainfall events or high humidity can promote a rapid deposition of pollen grains next to their production source, causing a low representation in the pollen rain records in faraway natural collectors of the populations of these plants (Silva 2007).
In general, moss polsters, as well as the bromeliad tanks that were palynologically surveyed by Gomes et al. (2014), are excellent natural air pollen collectors in Caatinga environments, provided that are present favorable conditions (moist microhabitats). As was highlighted in the preceding paragraphs, aspects related to floristic diversity, physiognomy and CBS pollination systems were viewed microscopically using the pollen spectra of the samples.

This study provides new information on the pollen records in moss polsters for Caatinga vegetation. Our results and future research on other areas of the Caatinga will shed light on the dynamics of pollen grains in an environment where palynological knowledge is scarce. This research is of substantial value for further paleoecological and paleoclimatic studies in semiarid Brazil.

\section{Acknowledgements}

We gratefully acknowledge the CAPES for the Masters scholarship to the first author, the Biodiversitas Foundation for allowing us to carry out our research at the CBS and the anonymous referees for their constructive comments. This study received financial support from the FAPESB and CNPq.

\section{References}

Ávila IR, Bauermann SG. 2001. Espectros de precipitação polínica durante as estações de outono-inverno no município de Novo Hamburgo, Rio Grande do Sul, Brasil. Pesquisas - série Botânica 51: 51-58.

Bagnouls F, Gaussen H. 1962. Estação seca e índice xerotérmico. Boletim Geográfico 20: 338-369.

Bauermann SG, Neves PCP. 1999. Espectros da chuva polínica na região de Canoas - Estação Caixa D’água. Pesquisas - série Botânica 49: 143-154.

Bauermann SG, Neves PCP, Giordani G, Ávila IR. 1998. Espectros de chuva polínica no município de Canoas/RS (Campus da ULBRA e arredores). Pesquisas - série Botânica 48: 193-205.

Bawa KS. 1990. Plant-pollinator interactions in tropical rain forests. Annual Review of Ecology and Systematics 21: 399-422.

Erdtman G. 1960. The acetolysis method. A revised description. Svensk Botanisk Tidskrift 39: 561-564.

Fontana SL. 2003. Pollen deposition in coastal dunes, south Buenos Aires Province, Argentina. Review of Paleobotany and Palynology 126: 17-37.

Forzza RC, Baumgratz JFA, Bicudo CEM, et al. 2010. Catálogo de plantas e fungos do Brasil Vol. 1. Rio de Janeiro, Andrea Jakobsson Estúdio \& Instituto de Pesquisas Jardim Botânico do Rio de Janeiro.

Gomes JMS, Lima LCL, Santos FAR, Silva FHM. 2014. First records of pollen rain in bromeliad tanks in a semi-arid area of Caatinga vegetation in Northeastern Brazil. Acta Botanica Brasilica 28: 176-183.

Grabandt RAJ. 1980. Pollen rain in relation to arboreal vegetation in the Colombian Cordillera Oriental. Review of Palaeobotany and Palynology 29: 65-147.

Hammer Ø, Harper DAT, Ryan PD. 2001. PAST: Palaeontological Statistics software package for education and data analysis. Palaeontologia Electronica 4: 9p. http://palaeo-electronica.org/2001_1/past/issue1_01. htm. 26 Jun. 2015.

Jones GD, Bryant VM Jr. 1996. Melissopalynology. In: Jansonius, J, McGregor DC. (eds.) Palynology: principles and applications. Salt Lake City, American Association of Stratigraphic Palynologists Foundations. p. 933-938.

Koff T. 2001. Pollen influx into Tauber traps in Estonia in 1997-1998. Review of Paleobotany and Palynology 117: 53-62. 
Koppler K, Vorwohl G, Koeniger N. 2007. Comparison of pollen spectra collected by four different subspecies of the honey bee Apis mellifera. Apidologie 38: 341-353.

Lazarova M, Petrova M, Jordanova M. 2006. Pollen monitoring in surface samples in mosses and pollen traps from the Beglika region (W Rhodopes). Phytologia Balcanica 12: 317-325.

McCune B, Grace J. 2002. Analysis of Ecological Communities. Gleneden Beach, MjM Software.

Montade V, Ledru MP, Burte J, et al. 2014. Stability of a Neotropical microrefugium during climatic instability. Journal of Biogeography 41: 1215-1226.

Moro MF, Lughadha EN, Filer DL, Araújo FS, Martins FR. 2014. A catalogue of the vascular plants of the Caatinga Phytogeographical Domain: a synthesis of floristic and phytosociological surveys. Phytotaxa 160: 001-118.

Pathirane L. 1975. Aerobiological literature in scientific periodicals. Grana 15: $145-147$.
Reese CA, Liu K. 2005. A modern pollen rain study from the central Andes region of the South America. Journal of Biogeography 32: 709-718.

Salgado-Labouriau ML. 1973. Contribuição à Palinologia dos Cerrados. Rio de Janeiro, Academia Brasileira de Ciências.

SEI - Superintendência de Estudos Econômicos e Sociais da Bahia. 2013. Informações geoambientais: informações geográficas e tipologia climática (Bahia). http://www.sei.ba.gov.br/side/frame_tabela. wsp?tmp.volta $=$ sg6\&tmp.tabela=t78. 21 Jul. 2015.

Silva FHM. 2007. Contribuição à palinologia das caatingas. PhD Thesis, Universidade Estadual de Feira de Santana, Brazil.

Vergamini SM, Valencia-Barrera RM, Sbersi F, Maffazzioli TF. 2006 Palinologia do componente herbáceo na atmosfera de Caxias do Sul, RS, Brasil. Acta Botanica Brasilica 20: 937-941.

Veriankaite L, Siljamo P, Sofiev M, Sauliene I, Kukkonen J. 2010. Modelling analysis of source regions of long-range transported birch pollen that influences allergenic seasons in Lithuania. Aerobiologia 26: 47-62. 